\title{
Post-Hackathon Learning Circles: Supporting Lean Startup Development
}

Tina Chan, MSc

Applied Health Sciences

University of Waterloo

Waterloo, ON N2L 3G1

Josephine McMurray, PhD Lazaridis School of Business

\& Economics

Wilfrid Laurier University

Brantford, ON N3T 3Y3

jmcmurray@wlu.ca

AnneMarie Levy, PhD

Lazaridis School of Business

\& Economics

Wilfrid Laurier University

Brantford, ON N3T 3Y3
Heidi Sveistrup, PhD

CEO, Chief Scientific Officer

Bruyère Research Institute

Ottawa, ON K1N 5C8

James Wallace, PhD

Applied Health Sciences

University of Waterloo

Waterloo, ON N2L 3G1

This is the author's version of the work. It is posted here by permission of ACM for your personal use. Not for redistribution. The definitive version was published in the Proceedings of the 2020 International Conference on Human Factors on Computing Systems on the ACM Digital Library at https://doi.org/10.1145/3334480.3375216

\begin{abstract}
Hackathons provide rapid, hands-on opportunities to explore innovative solutions to problems, but provide little support to teams in moving those solutions into practice. We explore the use of post-hackathon

Learning Circles to connect hackathon teams with key stakeholders, to reflect on prototypes and consider

business models. We conducted a qualitative field study with 4 post-hackathon teams on the theme of technology, social isolation, and aging. Our results show that Learning Circles are an effective way to involve stakeholders early in the development process, and to develop a deeper understanding of users, markets, and technology.
\end{abstract}

\section{Author Keywords}

Hackathon; Participatory Design; Social Isolation; Aging

\section{Introduction}

Hackathons are time-limited, hands-on opportunities for interested "hackers" to identify novel solutions to often intractable societal problems. They provide a competitive environment in which participants design develop, program, pitch and present a solution to a specified problem - often over 24 hours or a weekend [1]. They have been lauded for their authenticity and occasion for experimentation; yet are criticized as 
having a lack of substantive outputs, and offering few opportunities for reflection or expert feedback [2]. That is, hackathons place great emphasis on creativity and the development of new prototypes, at the expense of grounding that development in the needs of real people and realistic business models.

There is also substantial interest in taking advantage of the creativity and disruptive potential of ideas developed by individuals during hackathons and facilitating a transition from hackathon team to startup. One thought leader imagines "startup phases" [3]. The lean startup concept [4-6] uses agile methods and focuses on connecting prototypes with a business model, and iteratively checking to ensure that they are consistent with the needs of stakeholders. This model shares many key ideas with user-centred design (UCD) in $\mathrm{HCI}$ (e.g. [7]), such as involvement of stakeholders early and often, and iteratively developing ideas through phases like requirements gathering, design, and evaluation. But it is not clear how to best facilitate the transition from hackathon to startup in practice.

In this work, we explore the use of Post-Hackathon Learning Circles (LCs) [8] to connect hackathon teams with key stakeholders, reflect on prototypes and consider business models. The LC is grounded in Aboriginal ritual practice and culture [9] where listening was valued for its role in problem-solving. McBride and Good refer to LCs as a "group of people who come together to engage in dialogue about a common interest" where the process is marked by "equality and empowerment of all participants" [9]. The LC technique comprises four phases - reflection, learning, planning, and action - provides structure to a meeting, embodies the collective wisdom of a group, equity of participants, a sense of shared community, and allows participants to change a situation or behavior based on peer evaluation [10]. Importantly, LCs provide a structured mechanism by which hackathon teams, who may not be familiar with UCD processes, can interact with key stakeholders on equal ground, gather new requirements, and confirm validity of their proposed business model.

\section{Background}

The Waterloo Region (WR), located in southwestern Ontario, Canada, has been successful in creating a competitive ecosystem for software innovation and entrepreneurship [11]. While active regional innovation ecosystems employ numerous mechanisms to support rapid innovation, health hackathons are a relatively new phenomenon in the WR. Health hackathons bring clinicians, researchers, industry and community stakeholders together to collaborate and co-create innovative solutions to persistent problems in the health and social care system [12]. They are timelimited, often over multiple days, sometimes involve a theme or focus for solution generation, and generally involve a pitch event in the final hours to select a winner. Success at these hackathon events will often result in the winning pitch team being provided with cash or in-kind support to move the solution generated at the hackathon along the startup development timeline.

The growing inclusion of patients and other users in healthcare system evaluation and innovation is welldocumented [13]. It is less evolved in the development of health and medtech innovation [14].This case study documents the process of a post-hackathon co-design event that involved the use of participatory methods 


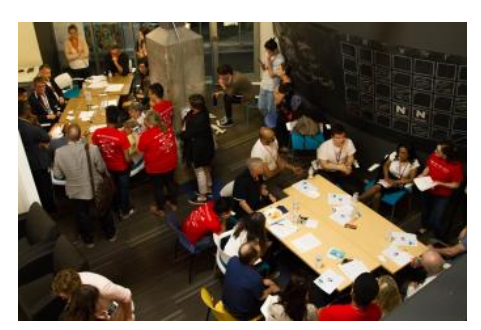

Figure 1. LCs as Part of the CoDesign Process

The teams and mentors networked over food, then the teams delivered a 5-minute pitch about their solutions and answered clarifying questions in a 3-minute question round with the judges and audience. Two pitch team members then participated in two codesign sessions over an hourlong period. Tables of up to twenty experts from a variety of disciplines and sectors provided feedback and engaged in generative discussion about the solutions. Facilitators documented ideas on chart paper and prompted discussion when appropriate. grounded in a LC [8], to provide insights from a multidisciplinary, multi-sectoral group of "mentors". Codesign is a construct that refers to "the effort to combine views, input and skills of people with many different perspectives to address a specific problem" [15]. The aim is to lower power and hierarchical barriers between stakeholders to allow them to actively engage with each other, and the problem, to identify real-life solutions [16]. The event followed a participatory approach to design [17] with a focus on strengthening the technology solutions with early insights from experts, particularly those with lived experience, and ensuring the involvement of marginalized voices in the re-imagining of solutions [18]. Not only did we hope to emancipate future users of proposed technology by facilitating early contact with it, but the goal was to influence, design and motivate the teams' continued interest in developing and commercializing their solutions.

\section{Method \& Co-Design Event}

The study leveraged a multi-city hackathon with the theme of "technology, social isolation and aging". Four Canadian teams from the Kitchener-Waterloo (KW) and Ottawa region Hacking Health Hackathons, pitched their solutions to a group of stakeholders, referred to as "mentors", at the co-design event held at the Communitech innovation hub in $\mathrm{KW}$. A summary of the four solutions is included in Table 1 . Mentors \& judges were recruited from the AGEWELL National Centre of Excellence and regional organizations representing municipal government, universities and colleges, local innovation hubs, health service organizations, industry, not-for-profits, advocates, and individuals with lived experiences.
The teams and mentors networked over food, then the teams delivered a 5-minute pitch about their solutions and answered clarifying questions in a 3-minute question round with the judges and audience. Two pitch team members then participated in two LCs over an hour-long period. LCs occurred at four tables focused on one hackathon team's idea (Figure 1). Up to twenty mentors from a variety of disciplines and sectors provided feedback and engaged in generative discussion about the solutions at each table (Table 2). Facilitators emphasized the goal of stimulating ideas with in-depth insights, and prioritizing appreciation over criticism. LC participants were invited to define a "desirable future state" for the presented projects.

A facilitator and notetaker at each table ensured that the discussion was balanced, progressive, and transcribed for the teams to review following the event. All participants received information about the event and their role in the co-design workshop ahead of time and were pre-assigned to a table and team so that they could pay attention to their pitch presentation.

We conducted semi-structured, half hour teleconference interviews with 12 of the 18 members of the pitch teams (Table 2): 2 women and 10 men, involved in the co-design workshop within two weeks of its occurring. Question prompts explored: their perceptions of the co-design sessions and LC related to the quality, type of feedback and insights from mentors; the type, quality and quantity of contacts and resources that were generated from the co-design and LC; the barriers and enablers to moving through the lean startup process. Interview data and field notes were transcribed verbatim, imported into NVivo version 11 , then iteratively coded [19] by three of the 
Abbreviations:

Hardware (HW)

Long-term care (LTC)

\begin{tabular}{|c|c|c|c|}
\hline Team & $\begin{array}{l}\text { 'Hack' } \\
\text { Type }\end{array}$ & Sector & $\begin{array}{c}\text { Start-Up } \\
\text { Stage }\end{array}$ \\
\hline 1 & $\begin{array}{l}\text { HW/ } \\
\text { App }\end{array}$ & $\begin{array}{l}\text { Health/ } \\
\text { Consumer }\end{array}$ & $\begin{array}{l}\text { Ideation: } \\
\text { User } \\
\text { Validation }\end{array}$ \\
\hline 2 & App & $\begin{array}{l}\text { Health/ } \\
\text { Retirement } \\
\text { Home/ } \\
\text { LTC }\end{array}$ & $\begin{array}{l}\text { Problem/ } \\
\text { Solution } \\
\text { Fit: } \\
\text { Market } \\
\text { Validation }\end{array}$ \\
\hline 3 & App & $\begin{array}{l}\text { Community } \\
\text { /Consumer }\end{array}$ & $\begin{array}{l}\text { Ideation: } \\
\text { Team } \\
\text { Building }\end{array}$ \\
\hline 4 & App & $\begin{array}{l}\text { Health/ } \\
\text { Rehab }\end{array}$ & $\mathrm{Va}$ \\
\hline
\end{tabular}

Table 1. Pitch teams' characteristics. researchers (JM, TC, JW) using an interpretive approach to identify common themes[19].

\section{Findings}

Three themes emerged from our analysis of the codesign and LC data: 1) Building a shared understanding; 2) Stakeholder culture clash; and 3) Mentor preferences.

\section{Building a Shared Understanding}

Market risk of new product (hardware and/or software) development is best mitigated with interdisciplinary teams and users iteratively engaged in different phases of the development life cycle $[20,21]$. One pitch participant and startup founder confirmed the value of involving stakeholders with new perspectives following a hackathon:

"There was quite a variety of people there from different walks of life... We were able to be able to come up a lot of different ideas." - ME

The pitch teams included members with differing roles, such as engineers, clinicians, and designers, and a variety of expertise, such as marketing, programming, and advanced mathematics. This diverse expertise helped build understanding in the LC:

"All of us were participating because we all had different expertise. If there are more technical person was there asking questions about that, the people that handle that aspect of it would engage with them."- MJ

To ensure equity, only two members of the pitch team assigned to their table were permitted to engage with the outside mentors. Any additional pitch team members were assigned as mentors to other teams' tables. This assignment helped to build networks of expertise between the pitch teams, but also contributed to understanding the potential for the product for other mentors:

"They had more developers on their team than we did...[and] considerably deeper understanding of various developing solutions and they actually helped us a lot with developing solutions or breaking apart...things that we can focus on and easier solutions"- NN

Other than by name, participants and mentors were not identified by role or expertise at the table. The goal was to influence non-judgmental and respectful interaction in the LC [22]:

"It is almost like equal opportunity to participate in the conversation I think." - JW

However, while beneficial to the breadth of feedback the number and variety of mentors at the table may have negatively influenced depth of discussion and all voices being heard:

"There were so many people to try to get through, I guess. I don't think there was too much time for any one person to have an in-depth conversation about it, but it was really good to get that broad range perspectives." - MJ

The LC facilitated a sprint, in the opinion of one software engineer participant. Their team's product had clinical applications, and the generative discussion from a broad range of subject matter expert mentors helped 


\begin{tabular}{|c|c|c|c|}
\hline \multirow{2}{*}{ Sector } & \multirow{2}{*}{ Total } & \multicolumn{2}{|c|}{ Gender } \\
\hline & & $\mathrm{M}$ & $\mathrm{F}$ \\
\hline Academia & 6 & 2 & 4 \\
\hline Community & 21 & 11 & 10 \\
\hline Government & 1 & 0 & 1 \\
\hline Health/Aging & 11 & 3 & 8 \\
\hline Industry & 20 & 15 & 5 \\
\hline $\begin{array}{l}\text { Research/ } \\
\text { Academia }\end{array}$ & 10 & 1 & 9 \\
\hline Total & 69 & 32 & 37 \\
\hline
\end{tabular}

Table 2. Sector representation of mentors, facilitators and judges at co-design event.

\begin{tabular}{lccc}
\hline & \multirow{3}{*}{ Total } & \multicolumn{2}{c}{ Gender } \\
Age (years) & & $\mathrm{M}$ & $\mathrm{F}$ \\
\hline $20-24$ & 2 & 2 & 0 \\
$25-29$ & 5 & 4 & 1 \\
$30-34$ & 4 & 3 & 1 \\
$35-39$ & 1 & 1 & 0 \\
unknown & 6 & 4 & 2 \\
\hline \multicolumn{1}{c}{ Total } & 18 & 14 & 4 \\
\hline Sector & & & \\
\hline Academia & 0 & 0 & 0 \\
Community & 0 & 0 & 0 \\
Government & 0 & 0 & 0 \\
Health/ & 7 & 5 & 2 \\
Aging & 6 & 4 & 2 \\
Industry & 5 & 5 & 0 \\
Research/ & 5 & 14 & 4 \\
Academia & Total & 18
\end{tabular}

Table 3. Pitch team members' demographics. their team to better understand actionable next steps for their product.

"There [were] discussions of in the future how you could do this and the discussion of the market area around it and discussion of the clinical applications and discussions from the health care providers perspective and the patients' perspective and things like that. I think it was a lot more hands-on and I think the discussion was a lot more useful." - RP

\section{Stakeholder Culture Clash}

The hackathon culture of socializing, solving problems and developing skills, has been critiqued as technological solutionism [18] and overly catering to male preferences $[23,24]$. Somewhat in contrast to this perspective, we observed the highly appreciative approach taken in judging at hackathons. Oftentimes the prize list is long, with many opportunities for recognition beyond the first place, in order to encourage teams to continue along the startup journey where recognition is currency to potential investors. LCs were designed using an appreciative approach [25] to avoid the critique of hyper-competition.

To support this notion, the LCs involved persons with lived experience, older adults living with or at risk of isolation, to recognize their role in co-creating more relevant products. However, the older adults were less encouraging of entrepreneurship and more focused on whether the solutions solved problems they understood:

"They were interested in solutions, but they weren't coming from a perspective of 'I made solutions for things before and I'm going to apply my experience here,' at least that's what I thought of it." - RP

Some participants felt their advice was tangential:

"I found that each session there was like a senior that would dominate everything and we wanted to be respectful of that person, so we wouldn't cut them off and say, "Let's move on to the next person or we're running out of time."

Nonetheless, the lived experience of the older participants was acknowledged as important:

"I think like elder adults. They do provide a lot of useful information for us because none of us really has a disease in knees, in other joints, so I think that part really help[ed] a lot." - JW

Many of the industry mentors provided feedback cast in positive language, consistent with their understandings of startup culture, and this motivated teams to consider commercializing their product:

"We talked about doing a startup with it, because we got a lot of positive feedback from the event... we talked to a bunch of different clinicians, people who run physio clinics, and stuff like that." - JP

Mentor Preferences

LCs invite persons of different backgrounds and skills from personal and professional experience to discuss topics with equality between all members [9]. Our case study included diversity in age and sector (Table 3), chosen based on attendance in the hackathon event, and the hackathon organizers' networks. Although 
equality was assumed in the LC model, teams felt some mentors' time was needed more than others. There was described value in deliberately spending time

networking outside of the LCs to receive feedback from a representative of a potential collaborator.

"It was hard because we met her doing the co-design, and we had to pay attention to the co-design and not necessarily just her." - AS

A reoccurring participant concern was the high number of mentors and limited time allotted for in-depth discussion at the LCs. It was also noted that having a participant list ahead of time might have allowed teams to prepare questions for specific mentors:

"Just knowing who might have been there...we might prepare ourselves to ask dumb questions. We have a lot of questions that we would love to get feedback on."

Although introductions were scheduled at the beginning of LCs, some tables would break into discussion prematurely, and teams were unaware of mentors' backgrounds:

"As they kept talking, you can guess, but it's-- I wasn't able to tell if someone was in a health profession or from the community right off the bat." - JC

\section{Discussion}

The HCI community recognizes the role of hackathons in learning and problem solving [27]. However, they require a complementary step, such as the LC, to meaningfully help move groups and their products along the startup development cycle.
LCs, as a post-hackathon event, can be an effective way to involve stakeholders early in the startup development process, and help hackers to develop a deeper understanding of the user, market, industry and technology related to their chosen problem. Our findings suggest that event logistics and pre-event assessment of mentor and participant expectations and familiarity with the startup process and culture would improve outcomes.

In considering the software development life cycle, the LC's reflecting and learning phases complement agile methods' steps of defining requirements for design [8] The LCs established a shared understanding between stakeholders and hackers to advance the development of feasible solutions to the problem of social isolation in older adults. Mentors with different perspectives and experience helped teams further define the problem space and form actionable steps to develop their solutions. The experience of communicating needs within interdisciplinary teams built critical teamwork skills needed in startups. Teams' knowledge of specific mentors attending before the event could also help them pre-plan appropriate questions in short session times if longer sessions are not feasible.

The role that future users play in helping to develop products that address market need led the authors to include many mentors from older adult communities. However, the authors were not sensitized to the lack of experience some of these mentors had with the rapidfire nature of the startup development process and the "appreciative rather than critical" role of mentors in it. Assessment of mentors' experience with hackathon culture and technology should be assessed in advance, and appropriate orientation provided to optimize 
mentors' experience and ability to support the startup ecosystem. In the words of a participant, "Mentorship [for mentors] leading up to the co-design sign finale would be very helpful."

\section{Conclusion \& What's next}

As an exploratory study, our case study describes a unique approach to the use of LCs after hackathons. LC's help to address commonly described shortcomings in the quality and type of feedback provided by mentors at hackathons. Furthermore, LCs build shared understanding that can support movement through the startup development cycle. The involvement of key end-users as mentors was an essential element of this LC, however a preparatory orientation for those unfamiliar with the hackathon culture would enrich the participant experience. Future research might explore whether an extended hackathon format and use of LCs might reduce development time, improve product acceptance by users, and increase the likelihood of forming a startup venture by following the teams longitudinally.

\section{Acknowledgements}

The authors thank AGEWELL, HackingHealth, Communitech, and SE Health for their support of this study.

\section{References}

[1] G. Briscoe and C. Mulligan, "Digital Innovation: The Hackathon Phenomenon," Creat. London Work.

Pap. No.6, no. 6, pp. 1-13, 2014.

[2] J. Warner and P. J. Guo, "Hack.edu: Examining how college hackathons are perceived by student attendees and non-attendees," ICER '17 Proc. 2017 ACM Conf. Int. Comput. Educ. Res., pp. 254-262, 2017.
[3] Startup Commons, "Startup development phases," Grow VC Operations: Startup Commons, 2015. [Online]. Available:

http://www.startupcommons.org/startupdevelopment-phases.html. [Accessed: 10-Oct2019].

[4] E. Ries, The Lean Startup, 1st ed. New York, N.Y.: Crown Publishing Group, 2011.

[5] A. Osterwalder and Y. Pigneur, Business Model Generation: A Handbook for Visionaries, Game Changers, and Challengers. Hoboken, New Jersey: John Wiley \& Sons, Inc., 2010.

[6] A. L. Dahlstrand and D. Politis, "Women business ventures in Swedish university incubators," Int. J. Gend. Entrep., vol. 5, no. 1, pp. 78-96, 2013.

[7] S. K. D. Dwivedi, S. Upadhyay, and A. K. Tripathi, "A working framework for the user-centered design approach and a survey of available methods," Int. J. Sceintific Res. Publ., vol. 2, no. 4, pp. 12-19, 2012.

[8] M. Riel, J. Rhoads, and E. Ellis, "Culture of critique: Online learning circles and peer reviews in graduate education," Self, Peer Gr. Assess. ELearning, pp. 142-168, 2006.

[9] J. McBride and J. Good, "Learning circles: What is their potential in Aboriginal community economic development?," Prince George, B.C., 2015.

[10] S. Wade and M. Hammick, "Action Learning in Theory and in Practice," Teach. High. Educ., vol. 4 no. 2, pp. 163-178, 1999.

[11] A. Bramwell, J. Nelles, and D. a. Wolfe, "Knowledge, innovation and institutions: Global and local dimensions of the ICT cluster in Waterloo, Canada," Reg. Stud., vol. 42, no. 1, pp. 101-116, 2008.

[12] C. Lee, "Health hackathons," Int. J. Infect. Dis., vol. 53, no. 2016, p. 7, 2016.

[13] Institute of Medicine, Patients charting the course: Citizen engagement in the learning health system. Washington, D.C.: The National Academies Press, 
2011

[14] H. McNeil, J. McMurray, K. Byrne, K. Grindrod, and P. Stolee, "Engagement of older adults in regional health innovation: The ECOTECH concept mapping project," Manuscr. Submitt. Publ., 2019.

[15] P. Bramwell, "Making the most of collaboration: An international survey of public service co-design," London, U.K., 2009.

[16] D. Szebeko and L. Tan, "Co-designing for society," Australas. Med. J., vol. 3, no. 9, pp. 580-590, 2010.

[17] S. Bardzell, "Utopias of participation: Feminism, design, and the futures," ACM Trans. Comput. Interact. - Spec. Issue Reimagining Particip. Des., vol. 25, no. 1, February 2018, pp. 1-24, 2018.

[18] A. Hope et al., "Hackathons as participatory design: Interating feminist utopias," in Proceedings of the $2019 \mathrm{CHI}$ Conference on Human Factors in Computing Systems - CHI '19, 2019.

[19] J. Corbin and A. L. Strauss, Basics of Qualitative Research: Techniques and Procedures for Developing Grounded Theory, Second., vol. 2. California, U.S.A.: Sage Publications Inc., 1998.

[20] E. Enkel, J. Perez-Freije, and O. Gassmann, "Minimizing market risks through customer integration in new product development: Learning from bad practice," Creat. Innov. Manag., vol. 14, no. 4, pp. 425-437, 2005.
[21] M. P. Lyndon et al., "Hacking hackathons: Preparing the next generation for the multidisciplinary world of healthcare technology " Int. J. Med. Inform., vol. 112, no. October 2017 pp. 1-5, 2018

[22] B. Granillo, R. Renger, J. Wakelee, and J. L. Burgess, "Utilization of the native American talking circle to teach incident command system to tribal community health representatives," J. Community Health, vol. 35, no. 6, pp. 625-634, 2010.

[23] S. Levy, Hackers: Heroes of the Computer Revolution. New York, USA: Dell Publishing, 2010.

[24] A. Decker, K. Eiselt, and K. Voll, "Understanding and improving the culture of hackathons: Think global hack local," Proc. - Front. Educ. Conf. FIE, vol. 2014, 2015

[25] E. Porter, C. Bopp, E. Gerber, and A. Voida, "Reappropriating hackathons: The production work of the CHI4Good Day of Service," Proc. 2017 CHI Conf. May 6-11, Denver, CO, pp. 810-814, 2017.

[26] M. Arena, R. Cross, J. Sims, and M. Uhl-Bien, "How to catalyze innovation in your organization," MIT Sloan Manag. Rev., vol. 58, no. 4, pp. 39-46, 2017.

[27] N. Taylor and L. Clarke, "Everybody's hacking: Participation and the mainstreaming of hackathons," Proc. CHI, pp. 1-12, 2018. 